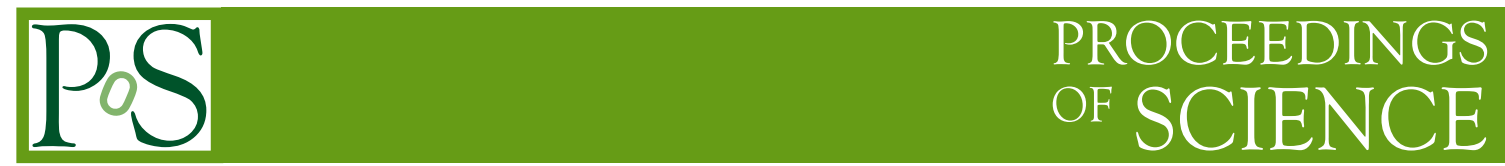

\title{
Spatial string tension revisited
}

\section{York Schröder*}

Faculty of Physics, University of Bielefeld, D-33501 Bielefeld, Germany

E-mail: yorksaphysik.uni-bielefeld.de

\section{Mikko Laine}

Faculty of Physics, University of Bielefeld, D-33501 Bielefeld, Germany

E-mail: laine@physik.uni-bielefeld.de

The spatial string tension, a classic non-perturbative probe for the convergence of the weakcoupling expansion at high temperatures, can be determined in full QCD as well as in a dimensionally reduced effective theory. Comparing both approaches, we find surprisingly good agreement almost down to the critical temperature of the deconfinement phase transition.

XXIIIrd International Symposium on Lattice Field Theory

25-30 July 2005

Trinity College, Dublin, Ireland

${ }^{*}$ Speaker. 


\section{Introduction}

The interest in QCD at temperatures $T$ larger than (a few) hundred $\mathrm{MeV}$ is triggered not only by purely theoretical reasons, but also by ongoing heavy ion collision experiments, and by cosmology. Given asymptotic freedom, a weak coupling expansion of this high-temperature phase seems well within reach. In practice, however, this expansion converges only slowly, and even shows a non-trivial analytic structure in the gauge coupling $g^{2}$.

By now, the problematic degrees of freedom have been identified. They are soft gauge-field modes with typical momenta $p \sim g T$, which give rise to odd powers in $g$, as well as ultrasoft modes $p \sim g^{2} T$, which enter the series via non-perturbative coefficients. For parametrically small values of the coupling $g$, these scales are well separated, such that an effective field theory treatment becomes feasible.

The general picture is that perturbation theory should work fine for parametrically hard scales $p \sim 2 \pi T$, while soft and ultrasoft scales need improved analytic schemes, or non-perturbative treatment. We will work within dimensionally reduced effective theories, in order to treat these different physical contributions separately, in a consistent scheme with controllable errors.

It appears mandatory to give quantitative evidence for the general picture sketched above. To this end, the strategy is to pick some simple observables and compare, as a function of $T$, full results (e.g. from $4 \mathrm{~d}$ lattice QCD simulations [1]) with predictions from the soft/ultrasoft effective theory setup, which should be exact for asymptotically large temperatures. This has been done for e.g. static correlation lengths [2], and in general agreement was found down to $T \sim 2 T_{\mathfrak{c}}$, where $T_{\mathfrak{c}}$ is the deconfinement phase transition temperature.

As another concrete example of an observable allowing for an unambiguous comparison, we discuss the spatial string tension $\sigma_{s}$ in this paper. It is defined in a manifestly gauge invariant way as the coefficient in the area law of a large rectangular Wilson loop $W_{s}\left(R_{1}, R_{2}\right)$ in $\left(x_{1}, x_{2}\right)$ plane,

$$
\sigma_{s} \equiv-\lim _{R_{1} \rightarrow \infty} \lim _{R_{2} \rightarrow \infty} \frac{1}{R_{1} R_{2}} \ln W_{s}\left(R_{1}, R_{2}\right) .
$$

It has been measured in SU(3) on the $4 \mathrm{~d}$ lattice, as a function of the temperature $T$ (e.g. Ref. [1]),

$$
\frac{\sqrt{\sigma_{s}}}{T}=\phi_{a}\left(\frac{T}{T_{\mathrm{c}}}\right)
$$

Our aim here is to get the effective theory prediction for $\sigma_{s}$, and to compare it with the lattice data, in order to assess the performance of the effective theory setup [3]. In the following two sections, we sketch the 2-step perturbative matching process of $4 \mathrm{~d}$ QCD onto $3 \mathrm{~d} \mathrm{M}$ (agnetostatic)QCD, and discuss convergence properties. In section 4 , we take existing data on $\sigma_{s}$ from $3 \mathrm{~d}$ lattice MQCD, match it to $4 \mathrm{~d} \mathrm{QCD}$, and compare with the $4 \mathrm{~d}$ lattice data.

\section{Effective theory setup: QCD $\rightarrow$ EQCD}

At high temperatures, all QCD dynamics is contained in a simpler, three-dimensional effective field theory called EQCD,

$$
\mathscr{L}_{\mathrm{E}}=\frac{1}{2} \operatorname{Tr} F_{k l}^{2}+\operatorname{Tr}\left[D_{k}, A_{0}\right]^{2}+m_{\mathrm{E}}^{2} \operatorname{Tr} A_{0}^{2}+\lambda_{\mathrm{E}}^{(1)}\left(\operatorname{Tr} A_{0}^{2}\right)^{2}+\lambda_{\mathrm{E}}^{(2)} \operatorname{Tr} A_{0}^{4}+\ldots,
$$


where $F_{k l}=i\left[D_{k}, D_{l}\right] / g_{\mathrm{E}}, D_{k}=\partial_{k}-i g_{\mathrm{E}} A_{k}$ with the dimensionful $3 \mathrm{~d}$ gauge coupling $g_{\mathrm{E}}$, and the dots represent higher-order operators. In order to correctly describe all contributions from hard and soft scales, the parameters of $3 \mathrm{~d}$ EQCD have to be regarded as matching coefficients, and are therefore related to the parameters of full QCD (being $g^{2}, T, N_{\mathrm{c}}, N_{\mathrm{f}}, \mu_{\mathrm{q}}, m_{\mathrm{q}}$ ). Perturbative matching [4] gives, schematically,

$$
\begin{aligned}
m_{\mathrm{E}}^{2} & =T^{2}\left\{\# g^{2}+\# g^{4}+\ldots\right\}, \\
\lambda_{\mathrm{E}}^{(1),(2)} & =T\left\{\# g^{4}+\# g^{6}+\ldots\right\}, \\
g_{\mathrm{E}}^{2} & =T\left\{g^{2}+\# g^{4}+\# g^{6}+\ldots\right\},
\end{aligned}
$$

where all coefficients symbolized by "\#” above are known. Most can be conveniently read from e.g. Ref. [5], while the $g^{6}$ term in the last line has been obtained only recently [3]. Higher-order coefficients could be obtained straightforwardly from the next order in the loop expansion.

There are also higher-order operators [6] in EQCD which become important at some point. In general, their relative magnitude can be estimated as [5]

$$
\delta \mathscr{L}_{\mathrm{E}} \sim g^{2} \frac{D_{k} D_{l}}{(2 \pi T)^{2}} \mathscr{L}_{\mathrm{E}} \sim g^{2} \frac{\left(g^{2} T\right)^{2}}{(2 \pi T)^{2}} \mathscr{L}_{\mathrm{E}}
$$

where we assumed to be considering an observable dominated by the ultrasoft scale $p \sim g^{2} T$. Thus, the relative magnitude is at most $\sim g^{6}$, smaller than any known terms in Eqs. (2.2)-(2.4).

At this point, having the first few terms of the perturbative series of, say, $g_{\mathrm{E}}^{2}=g_{\mathrm{E}}^{2}\left(g^{2}, T\right)$ at hand, one may ask about its convergence properties. In practice, renormalization is needed of course: let $g^{2}=g^{2}(\bar{\mu})$ be the (4d QCD) $\overline{\mathrm{MS}}$ coupling. From the solution of the 2-loop renormalization group equation, we define the $\overline{\mathrm{MS}}$ scale parameter as usual, and find the 2-loop running coupling as a function of $\bar{\mu} / \Lambda_{\overline{\mathrm{MS}}}$,

$$
\begin{aligned}
\Lambda_{\overline{\mathrm{MS}}} & \equiv \lim _{\bar{\mu} \rightarrow \infty} \bar{\mu}\left[b_{0} g^{2}(\bar{\mu})\right]^{-b_{1} / 2 b_{0}^{2}} \exp \left[-\frac{1}{2 b_{0} g^{2}(\bar{\mu})}\right], \\
\frac{1}{g^{2}(\bar{\mu})} & \approx 2 b_{0} \ln \frac{\bar{\mu}}{\Lambda_{\overline{\mathrm{MS}}}}+\frac{b_{1}}{b_{0}} \ln \left(2 \ln \frac{\bar{\mu}}{\Lambda_{\overline{\mathrm{MS}}}}\right),
\end{aligned}
$$

where $b_{0} \equiv-\beta_{0} / 2(4 \pi)^{2}, b_{1} \equiv-\beta_{1} / 2(4 \pi)^{4}$ are coefficients of the QCD beta function. Hence, we can now write $g_{\mathrm{E}}^{2}=g_{\mathrm{E}}^{2}\left(\bar{\mu}, \Lambda_{\overline{\mathrm{MS}}}, T\right)=T \phi_{b}\left(\bar{\mu} / T, T / \Lambda_{\overline{\mathrm{MS}}}\right)$ as a function of two dimensionless variables.

Formally, the renormalization scale dependence is of higher order, while numerically, there is $\bar{\mu}$ dependence due to our truncation of the perturbative series. We are free to choose some optimization procedure, e.g. the principle of minimal sensitivity, according to which we choose $\bar{\mu}_{\mathrm{opt}}$ as the extremum of the 1-loop expression for $g_{\mathrm{E}}^{2}$. This leaves us $g_{\mathrm{E}}^{2}=T \phi_{c}\left(T / \Lambda_{\overline{\mathrm{MS}}}\right)$ as a function of one variable only, which is plotted in the left panel of Fig. 1 for $N_{\mathrm{f}}=3$. Comparing 1-loop and 2-loop expressions (the gray band shows the effect of a scale variation within $\bar{\mu}=(0.5 \ldots 2.0) \times$ $\bar{\mu}_{\text {opt }}$, note that the process of perturbative matching shows very comforting convergence properties: corrections are in the 10-20\% range, and scale dependence gets significantly reduced.

In the right panel of Fig. 1, we show the effective gauge coupling $\alpha_{\mathrm{s}}^{\text {eff }} \equiv g_{\mathrm{E}}^{2} / 4 \pi T$ of EQCD, for several $N_{\mathrm{f}}$, in a much smaller temperature interval close to the phase transition temperature $T_{\mathrm{c}} \sim \Lambda_{\overline{\mathrm{MS}}}$. Noting that this $3 \mathrm{~d}$ effective coupling is reasonably small even at these low temperatures, we are led yet again to observe that treating the hard modes perturbatively appears well justified. 

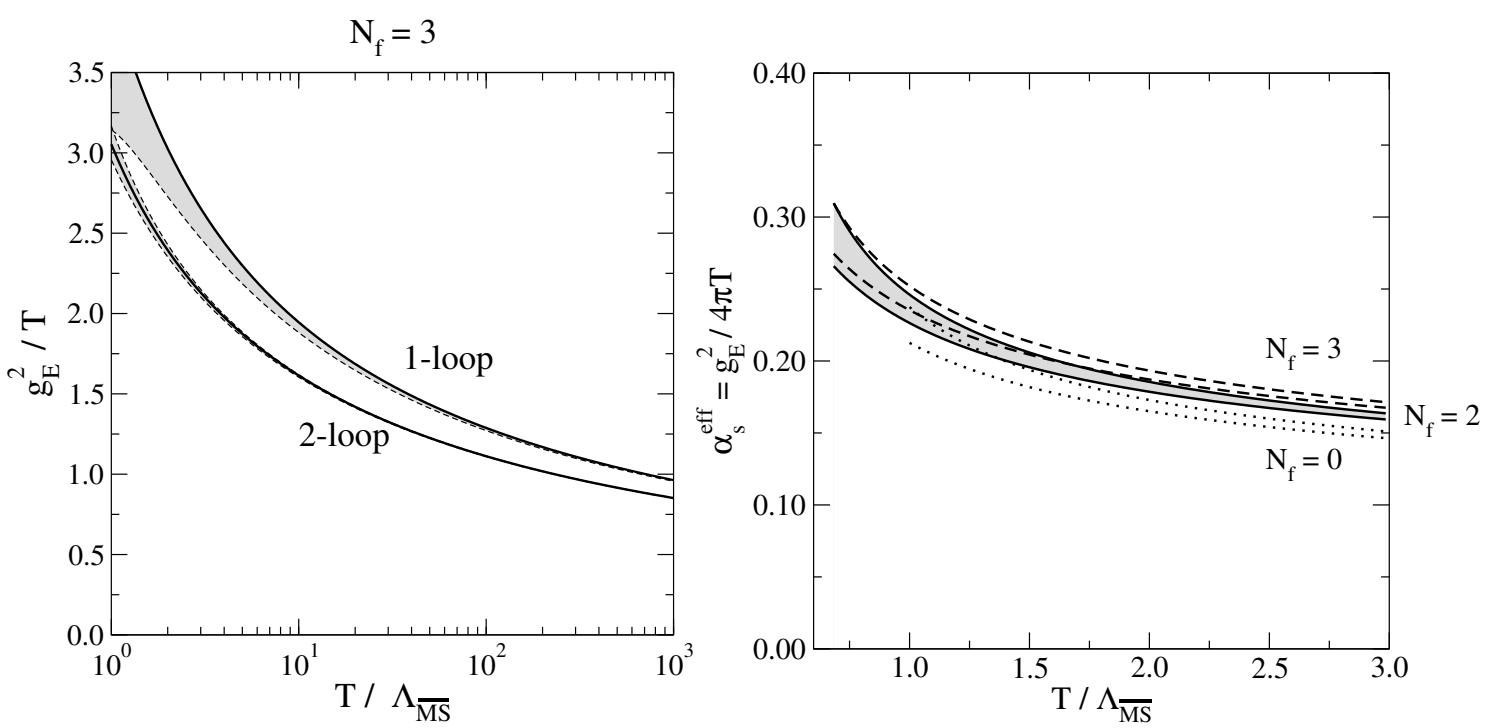

Figure 1: Left panel: The 1- and 2-loop values for $g_{E}^{2} / T$. For each $T$, the scale $\bar{\mu}$ has been fixed to $\bar{\mu}_{\text {opt }}$ as explained in the text. The gray band corresponds to a variation of $\bar{\mu}_{\text {opt }}$ within a factor of two. Right panel: The effective 2-loop gauge coupling of EQCD, $\alpha_{s}^{\text {eff }} \equiv g_{E}^{2} / 4 \pi T$, for various values of $N_{\mathrm{f}}$. Scale dependence results from $\bar{\mu}_{\text {opt }}$ variation as before. Note the different ranges of temperatures on the horizontal axes.

\section{Effective theory setup: EQCD $\rightarrow$ MQCD}

The low-energy behaviour of 3d EQCD is contained in another three-dimensional effective field theory, called MQCD,

$$
\mathscr{L}_{\mathrm{M}}=\frac{1}{2} \operatorname{Tr} F_{k l}^{2}+\ldots
$$

As before, the dots stand for higher-order operators, while the matching coefficients can be determined perturbatively $[7,3]$

$$
g_{\mathrm{M}}^{2}=g_{\mathrm{E}}^{2}\left\{1+\# \frac{g_{\mathrm{E}}^{2}}{m_{\mathrm{E}}}+\# \frac{g_{\mathrm{E}}^{4}}{m_{\mathrm{E}}^{2}}+\# \frac{g_{\mathrm{E}}^{2} \lambda_{\mathrm{E}}^{(1),(2)}}{m_{\mathrm{E}}^{2}}+\ldots\right\} .
$$

Let us note here - without showing the corresponding plot - that this expansion converges extremely well, even close to $T_{\mathrm{c}}$. Hence, we can safely ignore higher loop corrections for $g_{\mathrm{M}}^{2}$.

The higher-order operators of MQCD,

$$
\delta \mathscr{L}_{\mathrm{M}} \sim g_{\mathrm{E}}^{2} \frac{D_{k} D_{l}}{m_{\mathrm{E}}^{3}} \mathscr{L}_{\mathrm{M}} \sim g_{\mathrm{E}}^{2} \frac{\left(g^{2} T\right)^{2}}{m_{\mathrm{E}}^{3}} \mathscr{L}_{\mathrm{M}}
$$

give a relative contribution parametrically smaller than any of the known terms in Eq. (3.2), and will be neglected in the following.

\section{Results}

We are now in a position to write down the effective theory prediction for the spatial string tension $\sigma_{s}$, Eq. (1.1). The observable $\sigma_{s}$ exists not only in $4 \mathrm{~d} \mathrm{QCD}$, but also in 3d SU(3) gauge 


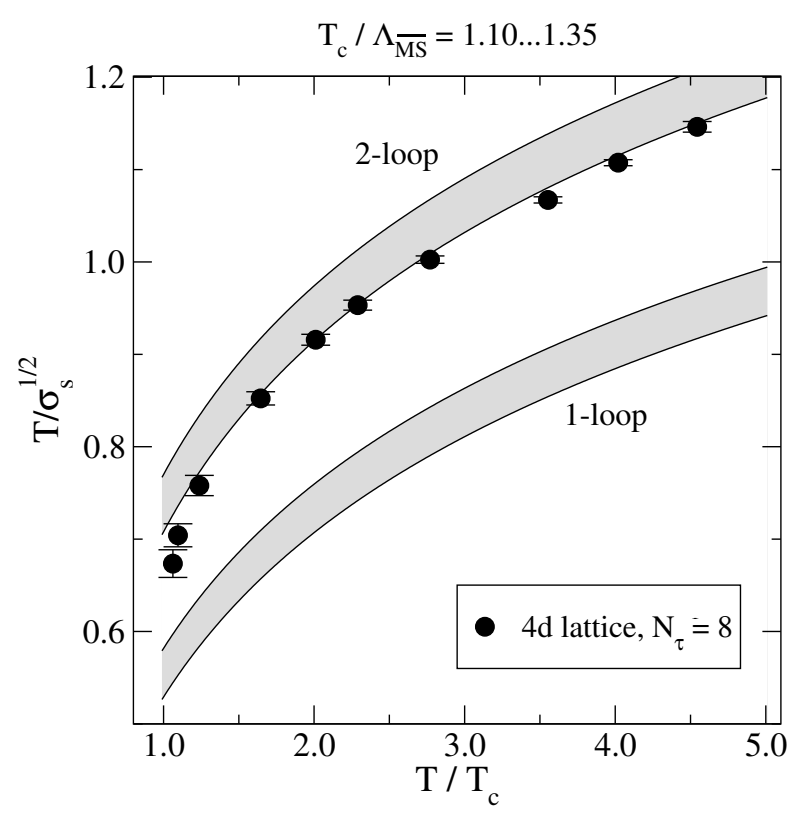

Figure 2: Comparison of 4d lattice data for the spatial string tension [1] with expressions obtained by combining 1-loop and 2-loop results for $g_{E}^{2}$ together with Eq. (3.2) and the non-perturbative value of the string tension of $3 d S U(3)$ gauge theory, Eq. (4.1). The upper edges of the bands correspond to $T_{\mathrm{c}} / \Lambda_{\overline{\mathrm{MS}}}=$ 1.35 , the lower edges to $T_{\mathrm{c}} / \Lambda_{\overline{\mathrm{MS}}}=1.10$.

theory, which is nothing but MQCD, Eq. (3.1). Since the $3 \mathrm{~d}$ gauge coupling is dimensionful, and furthermore is the only scale that MQCD possesses, naive dimensional analysis dictates $\sigma_{s}=\# g_{\mathrm{M}}^{4}$. The proportionality constant is non-perturbative, and can be measured by $3 \mathrm{~d}$ lattice simulations. Taking most recent lattice data [8],

$$
\frac{\sqrt{\sigma_{s}}}{g_{\mathrm{M}}^{2}}=0.553(1)
$$

To compare with the $4 \mathrm{~d}$ lattice results of the form shown in Eq. (1.2) (see Fig. 2), we need to relate $g_{\mathrm{M}}^{2}$ and $T$. First, using Eq. (3.2) and Eqs. (2.2)-(2.4),

$$
\frac{\sqrt{\sigma_{s}}}{T}=0.553(1) \frac{g_{\mathrm{M}}^{2}}{g_{\mathrm{E}}^{2}} \frac{g_{\mathrm{E}}^{2}}{T}=\phi_{d}\left(\frac{T}{\Lambda_{\overline{\mathrm{MS}}}}\right) .
$$

Next, we need to relate $\Lambda_{\overline{\mathrm{MS}}}$ and $T_{\mathrm{c}}$. This is in fact a classic problem in (4d) lattice QCD. One line of measurements [9] employs the $T=0$ string tension to get [10]

$$
\frac{T_{\mathrm{c}}}{\Lambda_{\overline{\mathrm{MS}}}}=\frac{T_{\mathrm{c}} / \sqrt{\sigma}}{\Lambda_{\overline{\mathrm{MS}}} / \sqrt{\sigma}}=1.16(4),
$$

while another possibility is to go via the Sommer scale [11]

$$
\frac{T_{\mathrm{c}}}{\Lambda_{\overline{\mathrm{MS}}}}=\frac{r_{0} T_{\mathrm{c}}}{r_{0} \Lambda_{\overline{\mathrm{MS}}}}=1.25(10) \text {. }
$$

To be conservative, we will consider the interval $T_{\mathrm{c}} / \Lambda_{\overline{\mathrm{MS}}}=1.10 \ldots 1.35$, which also incorporates the result of Ref. [12]. 
In Fig. 2, we finally compare the $3 \mathrm{~d}$ effective theory prediction for $\sigma_{s}$ (gray bands) with the $4 \mathrm{~d}$ lattice data (black dots). As a caveat, note that the lattice data has not been extrapolated to the continuum limit. On the other hand, we stress that the comparison is parameter-free. We may take the excellent agreement of the 2-loop prediction with the lattice data as support for hard/soft+ultrasoft picture of thermal QCD.

To conclude, we have given yet another example of a static observable in thermal QCD, for which the program of dimensional reduction works well, even down to temperatures $T \sim 2 T_{\mathrm{c}}$.

\section{References}

[1] G. Boyd, J. Engels, F. Karsch, E. Laermann, C. Legeland, M. Lütgemeier and B. Petersson, Thermodynamics of SU(3) Lattice Gauge Theory, Nucl. Phys. B 469 (1996) 419 [hep-lat/9602007].

[2] M. Laine and O. Philipsen, The non-perturbative QCD Debye mass from a Wilson line operator, Phys. Lett. B 459 (1999) 259 [hep-lat/9905004]; A. Hart and O. Philipsen, The spectrum of the three-dimensional adjoint Higgs model and hot SU(2) gauge theory, Nucl. Phys. B 572 (2000) 243 [hep-lat/9908041]; A. Hart, M. Laine and O. Philipsen, Static correlation lengths in QCD at high temperatures and finite densities, Nucl. Phys. B 586 (2000) 443 [hep-ph/0004060].

[3] M. Laine and Y. Schröder, Two-loop QCD gauge coupling at high temperatures, JHEP 03 (2005) 067 [hep-ph/0503061].

[4] E. Braaten and A. Nieto, Free energy of QCD at high temperature, Phys. Rev. D 53 (1996) 3421 [hep-ph/9510408].

[5] K. Kajantie, M. Laine, K. Rummukainen and M. Shaposhnikov, $3 d S U(N)+$ adjoint Higgs theory and finite-temperature $Q C D$, Nucl. Phys. B 503 (1997) 357 [hep-ph/9704416]; K. Kajantie, M. Laine, K. Rummukainen and Y. Schröder, The pressure of hot QCD up to $g^{6} \ln (1 / g)$, Phys. Rev. D 67 (2003) 105008 [hep-ph/0211321].

[6] S. Chapman, A New Dimensionally Reduced Effective Action For QCD At High Temperature, Phys. Rev. D 50 (1994) 5308 [hep-ph/9407313].

[7] P. Giovannangeli, Two loop renormalization of the magnetic coupling in hot QCD, Phys. Lett. B 585 (2004) 144 [hep-ph/0312307]; Two loop renormalization of the magnetic coupling and non-perturbative sector in hot QCD, hep-ph/0506318.

[8] M.J. Teper, $S U(N)$ gauge theories in 2+1 dimensions, Phys. Rev. D 59 (1999) 014512 [hep-lat/9804008]; B. Lucini and M. Teper, $S U(N)$ gauge theories in 2+1 dimensions: Further results, Phys. Rev. D 66 (2002) 097502 [hep-lat/0206027].

[9] B. Beinlich, F. Karsch, E. Laermann and A. Peikert, String tension and thermodynamics with tree level and tadpole improved actions, Eur. Phys. J. C 6 (1999) 133 [hep-lat/9707023].

[10] B. Lucini, M. Teper and U. Wenger, The high temperature phase transition in $S U(N)$ gauge theories, JHEP 01 (2004) 061 [hep-lat/0307017]; G.S. Bali and K. Schilling, Running coupling and the Lambda parameter from SU(3) lattice simulations, Phys. Rev. D 47 (1993) 661 [hep-lat/9208028].

[11] S. Necco, Universality and scaling behavior of RG gauge actions, Nucl. Phys. B 683 (2004) 137 [hep-lat/0309017]; S. Capitani, M. Lüscher, R. Sommer and H. Wittig [ALPHA Collaboration], Non-perturbative quark mass renormalization in quenched lattice QCD, Nucl. Phys. B 544 (1999) 669 [hep-lat/9810063].

[12] S. Gupta, A precise determination of $T_{\mathrm{c}}$ in $Q C D$ from scaling, Phys. Rev. D 64 (2001) 034507 [hep-lat/0010011]. 